\title{
A MULTIPROCESSOR SYSTEM FOR SIMULATION OF MULTISENSOR DISTRIBUTED DECISION ALGORITHMS
}

\author{
Charles $W$. Therrien \\ Mark A. Schon \\ Sung Chu Hahn \\ Naval Postgraduate School \\ Monterey, California 93943
}

\begin{abstract}
Distributed decision problems arise when two or more sensors viewing the same phenomenon must work cooperatively to draw inferences about the observed situation. Typical examples are in target detection and target classification. Such problems are characterized by distributed processing of information and communication between processors over a limited bandwidth data link. This paper presents some statistical distributed decision algorithms and describes their implementation on a set of loosely coupled multiprocessor clusters which simulate the distributed environment characterizing multisensor decision problems.
\end{abstract}

\section{INTRODUCTION}

There are a number of situations where where two or more sensors are observing the same phenomenon with the purpose of drawing inferences about the situation being observed. A common example is that of radars observing targets for purposes of detection and/or classification. This kind of scenario is illustrated in Fig. 1. It is important that the information acquired by the two sensors be processed jointly in order to arrive at the best decision. Simple processing of the data by each individual sensor and reporting of the results to a higher level intelligent process for arbitration is not necessarily the best procedure. A simple example can be constructed of two sensors observing a common air space in order to make a binary decision: $H_{1}$ : a target is present or $H_{0}$ : no target is present. Situations arise when the optimal individual decisions are both $H_{1}$ while the optimal joint decision is $H_{0}$. Thus a "correct" decision cannot be made about the existence of a target by any higher level process unless the distributive nature of the problem is built into the front end statistical decision procedure.

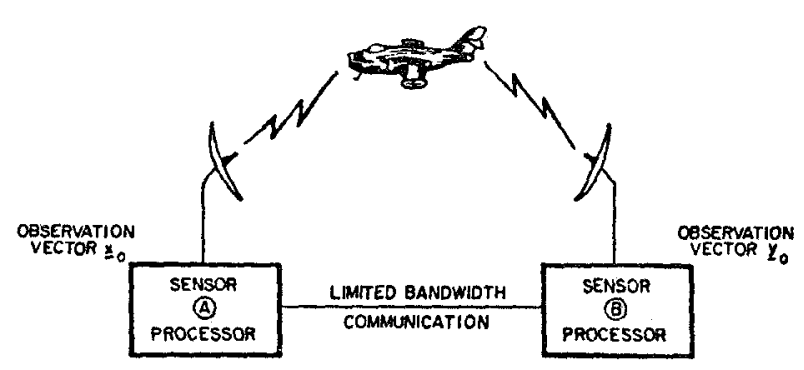

Fig. 1 - Distributed decision scenario

In this paper we are concerned with distributed processing of the observation data to achieve optimal or near optimal decisions. The sensors are assumed able to perform computations to partially reduce the raw observation data and are able to cornmunicate among themselves over a limited bandwidth channel. Algorithms that operate under these constraints are referred to as distributed decision algorithms. Algorithms that would perform the decision by 
sending all of the obserration data to a central fusion point for processing are referred to as centralized algorithms. Centralized algorithms are not of concern here except in providing a performance standard to which the distributed algorithms can be compared.

This paper contains a discussion of some specific distributed decision algorithms and the implementation of one of these in a distributed multiprocessor system that simulates two interacting sensors. Issues of process organization and synchronization that are crucial to the distributed processing of the observed data are discussed in detail.

\section{DISTRIBUTED ALGORITHMS}

This section contains a discussion of some alternative approaches to a. simple binary (two hypothesis) decision problem. The various algorithms have overall similar characteristics in that local computations are performed by each sensor, reduced data. is exchanged over a limited capacity cornmunications channel, and final decisions are made based on the joint observations of the sensors.

The discussion here assumes that there are only two sensors involved (A and B) and that the task is to make a binary decision $\left(H_{1}\right.$ or $\left.H_{0}\right)$. Generalization of most of these methods to multiple sensors and/or multiple hypotheses is possible.

\section{Tenney - Sandell Procedure}

Tenney and Sandell 1 seem to have been the first to look at distributed decision algorithms of the type described here. In their work the observations of the two sensors were assumed independent when conditioned on the hypotheses. Such independence of observations could arise if the sensors measured physically different parameters of the target (e.g. radar cross section and infrared radiation). The sensors were to each make a binary decision based on their own observations and send the result (a single bit) to a fusion center for arbitration. A cost criterion was devised that depended on the decisions made by each sensor and on the two hypotheses. Tenney and Sandell showed that the procedure that minimized the expected value of the cost is a likelihood ratio test at each sensor. How- ever. the thresholds used by the two sensors are coupled through some integral equations.

\section{Relaxation Algorithms}

Relaxation algorithms $[2,3]$ are another way to execute distributed decisions. These algorithms are less well-founded in a theoretical sense, but seem to work well in practice. In relaxation, each sensor makes an initial decision based on its own observations. The decisions are exchanged and each sensor may then revise its decision based on the new information. The procedure works best when there are multiple decision makers involved and may require more than a single iteration to converge.

\section{Algorithms Based on a Generalized Likelihood Ratio Test}

If the information exchanged between sensors is more than a single bit, but limited to, say, a single floating point number, then a whole new class of procedures suggest themselves. In particular, if the observations are independent as in the TenneySandell analysis, then the likelihood ratio for the joint observations factors into two parts, each depending only on the observations of a single sensor. Thus each sensor can compute the likelihood ratio (or $\log$ likelihood ratio) statistic for its own observations and send it to the other sensor. Each sensor then has the complete information required for making a decision to minimize probability of error based on the joint observations.

A more interesting problem occurs if the observations are correlated. In this case the joint likelihood ratio does not factor in such a convenient way. However, a procedure can be suggested that leads to a relatively simple decision algorithm. Let the observations acquired by sensors $A$ and $B$ be represented by $\mathbf{x}_{0}$ and $\mathbf{y}_{0}$ respectively. The optimal centralized test to minimize the probability of error has the form

$$
\left.\ln \frac{p_{1}\left(\mathbf{x}_{0}, \mathbf{y}_{0}\right)}{p_{2}\left(\mathbf{x}_{0}, \mathbf{y}_{0}\right)}=\ln \frac{p_{1}\left(\mathbf{x}_{0}\right)}{p_{2}\left(\mathbf{x}_{0}\right)}+\ln \frac{p_{1}\left(\mathbf{y}_{0} \mid \mathbf{x}_{0}\right)}{p_{2}\left(\mathbf{y}_{0} \mid \mathbf{x}_{0}\right)}\right)_{H_{0}}^{H_{1}} \ln T
$$


where the subscript $i$ on the probability density function $p$ indicates that the density function is for $H_{i}$.

A distributed form of this tesi can be developed by allowing $A$ to compute the first term in (1) and allowing B to compute an approximation to the second term (the conditional log likelihood ratio) by using some estimate for the observations $\mathbf{x}_{0}$. This procedure is known as a generalized likelihood ratio test [4]. In essence, when the density function involves an unknown parameter (in our case $\mathbf{x}_{0}$ ) an estimate is made based on each hypothesis and used in the corresponding density function. If $B$ sends the result of this computation to $A$ then the test (1) can be evaluated to make a decision. A symmetric computation can be made with the roles of $A$ and $B$ reversed.

The decision rule just described has a number of essential differences from the corresponding centralized algorithm. First, since the likelihood ratio evaluated by one sensor uses an estimate for the other sensor's observations, the performance of the algorithm will in general be different and suboptimal when compared to the centralized test. Second, since the two sensors perform symmetric computations with the roles of $\mathbf{x}_{0}$ and $\mathbf{y}_{0}$ reversed, there will, in general be a region of the combined observation space where the decisions of the two sensors do not agree.

The properties of this class of distributed decision algorithms is dependent on the various methods of estimating the unknown observations $\mathbf{x}_{0}$. If the sensors are allowed to interchange only a single statistic then the estimate for $\mathbf{x}_{0}$ must be derived entirely from $y_{0}$ (e.g. using MAP estimation) and the resulting decision rule is of the form

$$
\lambda_{A}\left(\mathbf{x}_{0}\right)+\lambda_{B}\left(\mathbf{y}_{0}\right)_{\substack{H_{0} \\ H_{0}}}^{H_{1}} \ln T
$$

This limits the degree to which the distributed test can approximate the centralized test since in many cases the centralized test will not be separable.

\section{Decision Based on the Nearest Neigh- bor Rule}

A final form of distributed decision algorithm is based on the k-nearest neighbor rule of pattern recognition [5]. In this nonparametric decision rule, a set of observations to be tested is represented as a point in a multidimensional observation space. Also existing in this space are previously given sets of points (training data) corresponding to each of the two hypotheses. The distance of the measured observations to each of the other points is computed to determine its $\mathrm{k}$ nearest neighbors. If most of the neighbors correspond to $H_{1}$ then the given observations are also associated with $H_{1}$, otherwise the given observations are classified according to $H_{0}$.

A distributed form of this decision rule can be developed by letting each sensor determine a small number of nearest neighbors in the $\mathbf{x}$ or $\mathbf{y}$ subspace. If the labels of these points and their distances from the observation data are interchanged, one can compute the distances in the xy observation space and classify the observation data. This policy does not guarantee that the true nearest neighbors will always be found but allows a decision to be made without further iterations and exchange of information.

\section{DISTRIBUTED MULTIPROCESSOR IMPLEMENTATION}

\section{Multiprocessor Environment}

The test environment for the distributed decision algorithms consists of a highly modular hardware base and a highly flexible software operating system. The hardware consists of two clusters of single board computers (SBC's), each sharing a common backplane and a local area network (LAN) serving as the communication link.

A cluster is configured with three SBC's, each with 64K RAM of local memory. An additional shared memory board is used for transfer of user data and 
system synchronization data over the LAX.

The operating system is a distributed multicomputer real-time executive 6 . It allows for asynchronous operation of processes resident on SBC"s in the same cluster and in separate clusters linked via the LAN.

Since this system models two sensors observing the same target at the same time , it is crucial that the computations of the two sensors be properly synchronized. One purpose of the implementation was to investigate this synchronization problem in the context of a typical algorithm. Synchronization was achieved by certain process control mechanisms available in the operating system. These mechanisms, referred to as primitives, are a type of semaphore and are typical of those available in many modern real-time operating systems.

The process control mechanisms are based upon a synchronization model which is event oriented. Processes coordinate various activities by signaling and observing events via synchronization variables known as eventcounts and sequencers.

An eventcount is a variable created upon user request and initialized with a value of zero. It is incremented by one each time the event associated with it occurs. The mechanism to signal this occurrence is a call to a system primitive called advance. A call to another system primitive, the await. causes the process to wait until the designated eventcount has reached a designated threshold.

A sequencer is associated with a shared resource such as the $\mathrm{LAN}$. It is a positive integer number generator which begins with the number zero. It increments by one after supplying the current number to any requesting process which wants to use the shared resource. The requesting process then waits until the eventcount. which tracks the present user of the shared resource. has been incremented to a value equal to the number obtained. When this occurs, the requesting process is able to use the resource. is referred to as a ticket operation. The mechanism used to obtain a number from the sequencer is referred to as a ticket operation.
The kernel of the operating system resident on each $\mathrm{SBC}$ schedules processes for execution. A process runs until it invokes one of the primitives (advance or await ), which results in the actions described above. System synchronization is accomplished by the user processes using system eventcounts and sequencers to control the orderly flow of computation.

\section{Algorithm Implementation}

The algorithm implemented as the test case was the one based on the generalized likelihood ratio test described earlier. Each microcomputer cluster can be viewed as a set of local processors for a particular sensor which obtains large volumes of raw data for initial processing. Parameters for the decision rule are loaded at system initialization from local disk storage. The raw observation data is then read and used to generate, in parallel, reduced partial results for both the local and remote clusters. One processor in the cluster performs the computations necessary to reduce the data from one file to the required statistics for local use. A second processor performs the parallel computations necessary to reduce the data from another file to the statistics required for the remote cluster.

Processes P2 and P3, residing on SBC's in the same cluster, perform the computations shown in Fig. 2

\section{SENSOR A}

\section{P2: Compute $\lambda_{A}\left(\mathrm{x}_{0}\right)$ Compute $\lambda_{A}\left(\mathbf{x}_{0}\right)+\lambda_{B}\left(\mathbf{y}_{0}\right)$}

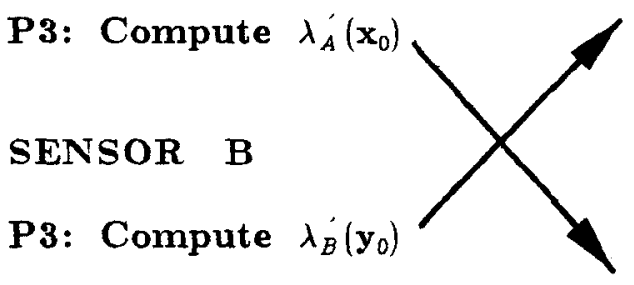

P2: Compute $\lambda_{B}\left(y_{0}\right)$ Compute $\lambda_{B}\left(\mathbf{y}_{0}\right)+\lambda_{A}^{\prime}\left(\mathbf{x}_{0}\right)$

Fig. 2 - Processes and computations 
Symmetric sets of computations are performed at sensors $\mathbf{A}$ and $\mathbf{B}$. in the same time frame, using the observations $\mathbf{x}_{0}$ and $\mathbf{y}_{0}$ respectively. Results are computed in processes $P 2$ and $P 3$ yielding the statistics shown. $\lambda_{A}\left(\mathbf{x}_{0}\right)$ and $\lambda_{B}\left(\mathbf{y}_{0}\right)$ are the reduced statistics used in further local computation and $\lambda_{A}^{\prime}\left(\mathbf{x}_{0}\right)$ and $\lambda_{B}^{\prime}\left(\mathbf{y}_{0}\right)$ are the statistics sent to the opposite sensors for further computation.

The statistic to be transferred is stored in shared memory and a ticket is obtained from the system sequencer. This places the request for user data to be transferred in a queue. Once this request is at the head of the queue, the system driver forms a packet and sends it to the remote cluster address indicated. The local process which produced the data subsequently advances an eventcount. That eventcount is sent to the other cluster by a procedure similar to the one for sending data. This causes the eventcount to be updated at the remote cluster following the arrival of the data. Once the eventcount is updated at the remote sensor, it allows a previously set await state to be satisfied and the process uses the data to continue its computations.

In the final stage of computation $\lambda_{A}\left(\mathbf{x}_{0}\right)$ and $\lambda_{B}\left(\mathbf{y}_{0}\right)$ the reduced statistics retained locally and $\lambda_{B}\left(\mathbf{y}_{0}\right)$ and $\lambda_{A}^{\prime}\left(\mathbf{x}_{0}\right)$ the statistics received from the remote sensor are added in process $\mathrm{P} 2$ of each sensor and compared to a threshold (see Figure 2). Results of the threshold decision are tabulated on the local consoles of each processor and the loop repeats with the next set of observations.

\section{CONCLUSIONS}

Distributed decision procedures allow cooperating sensors to produce more reliable decisions than are possible when each sensor acts alone. Such decision procedures are characterized by the need to perform local computations at each sensor and to communicate partial results to the other sensor. Several different types of algorithms can be developed.

A typical distributed decision algorithm was implemented on a set of multiprocessors connected via a local area net- work. Each node of the network contained a cluster of identical processors sharing some common local resources. The implementation required intercluster as well as intracluster synchronization of events to ensure the timely input of observation data and coordinated computation using data shared between clusters. The implementation demonstrated the feasibility of performing distributed decision algorithms in a realistic environment and underscored the need for carefully designed network-wide process control mechanisms.

\section{Acknowledgement}

This work was sponsored by Navy project NAVSEATASK PMS-400-85-750-1.

\section{References}

[1] R.R. Tenney and N.R. Sandell, "Detection with distributed sensors", IEEE Trans. Aerospace and Electronic Systems, Vol. AES-17, No.4, July 1981

[2] A.Rosenfeld, R.A. Hummel, and S.W. Zucker, "Scene Labeling by Relaxation Operations", IEEE Trans. Systems, Man, and Cybernetics, Vol. 6, 1976 pp 420-433

3 R.M. Haralick, "Decision Making in Context". IEEE Trans. Pattern Anal. Machine Intelligence, Vol. PAMI-5, No.4, July 1983

4. H.L. Van Trees, Detection Estimation and Modulation Theory, Part I (John Wiley \& Sons, New York, 1968)

5 R.O. Duda and P.E. Hart, Pattern Classification and Scene Analysis, (John Wiley \& Sons, New York, 1973)

6. D.J. Brewer, A Real-Time Executive for Multiple-Computer Clusters, M.S. Thesis, Naval Postgraduate School, Monterey, California, December 1984 